\title{
EFECTOS DE LA TEMPERATURA EN LA GERMINACIÓN DE SEMILLAS DE LAS ESPECIES PROSOPIS PALLIDA (HUMB. \& BONPL. EX WILLD.) KUNTH Y PROSOPIS JULIFLORA (SW.) DC
}

\author{
AUTORES: $\quad$ Marcos Pedro Ramos-Rodríguez ${ }^{1}$ \\ Jhonny Lenin Ugalde-Párraga ${ }^{2}$ \\ Jenniffer Alexandra Medranda-Mendieta ${ }^{3}$ \\ Ignacio Estévez-Valdés ${ }^{4}$ \\ Tayron Omar Manríquez-Toala ${ }^{5}$
}

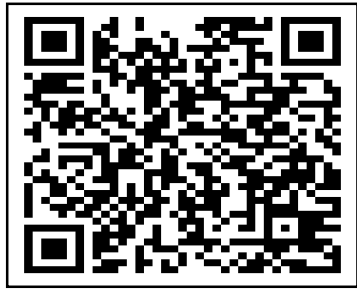

\section{DIRECCIÓN PARA CORRESPONDENCIA: (marcos.ramos@unesum.edu.ec)}

Fecha de recepción: 10/04/2021

Fecha de aceptación: 21/09/2021

\section{RESUMEN}

Prosopis pallida (Humb. \& Bonpl. ex Willd.) Kunth y Prosopis juliflora (Sw.) DC., son dos de las especies arbóreas de mayor importancia económica y ecológica en las zonas áridas y semiáridas del mundo. Las especies de Prosopis han llamado la atención por su gran capacidad para sobrevivir en ambientes muy inhóspitos. Este trabajo tuvo el objetivo de evaluar los efectos de la temperatura en la germinación de las especies mencionadas como un indicador de su respuesta al fuego. Se trabajó con 40 tratamientos resultantes del arreglo trifactorial $2 \times 5 \times 4+1$, (dos especies, cinco valores de temperaturas, cuatro tiempos de exposición y un testigo), los cuales se asignaron a un diseño experimental completamente al azar con tres repeticiones. La normalidad de los datos se comprobó con la prueba de Shapiro-Wilks. La comparación de las medias se realizó con la prueba $t$ de Student para la viabilidad y con un análisis de varianza y la prueba de Tukey para la diferencia entre pares de medias para el porcentaje de germinación. Se trabajó con un nivel de significancia del $0,05(\mathrm{p}=0,05)$. El análisis estadístico se realizó con el programa SPSS Statistics for Windows (Version 22.0). El porcentaje de germinación de las semillas utilizadas fue afectado tanto por el aumento de la temperatura como por el tiempo de exposición. No obstante, pudieran germinar después de la ocurrencia de fuegos de baja intensidad que generen temperaturas de hasta 80 y $100{ }^{\circ} \mathrm{C}$ para $P$. pallida y $P$. juliflora, respectivamente.

PALABRAS CLAVE: Viabilidad de las semillas; germinación; respuesta al fuego.

\footnotetext{
${ }^{1}$ Universidad Estatal del Sur de Manabí, Ecuador. E-mail: marcos.ramos@unesum.edu.ec

${ }^{2}$ Universidad Estatal del Sur de Manabí, Ecuador. E-mail: jhonnylup@hotmail.com

${ }^{3}$ Universidad Estatal del Sur de Manabí, Ecuador. E-mail: smileever25@outlook.es

${ }^{4}$ Universidad Estatal del Sur de Manabí, Ecuador. E-mail: ignacio.estevez@unesum.edu.ec

${ }^{5}$ Universidad Estatal del Sur de Manabí, Ecuador. E-mail: tyron.manrique@unesum.edu.ec
} 
Ramos-Rodríguez, M. P., Ugalde-Párraga, J. L., Medranda-Mendieta, J. A., Estévez-Valdés, I., Manríquez-Toala, T.

\title{
EFFECTS OF TEMPERATURE ON THE GERMINATION OF SEEDS OF THE SPECIES PROSOPIS PALLIDA (HUMB. \& BONPL. EX WILLD.) KUNTH AND PROSOPIS JULIFLORA (SW.) DC
}

\begin{abstract}
Prosopis pallida (Humb. \& Bonpl. Ex Willd.) Kunth and Prosopis juliflora (Sw.) DC., are two of the tree species of greatest economic and ecological importance in the areas arid and semi-arid of the world. Prosopis species have attracted attention for their great ability to survive in very inhospitable environments. This work had the objective of evaluating the effects of temperature on the germination of the mentioned species as an indicator of their response to fire. We worked with 40 treatments resulting from the $2 \times 5 \times 4+1$ trifactorial arrangement, (two species, five temperature values, four exposure times and one control), which were assigned to a completely randomized experimental design with three repetitions. The normality of the data was checked with the Shapiro-Wilks test. The comparison of the means was carried out with the Student's t test for viability and with an analysis of variance and the Tukey test for the difference between pairs of means for the germination percentage. We worked with a significance level of 0.05 $(\mathrm{p}=0.05)$. Statistical analysis was performed with the SPSS Statistics for Windows program (Version 22.0). The germination percentage of the seeds used was affected both by the increase in temperature and by the exposure time. However, they could germinate after the occurrence of low intensity fires that generate temperatures of up to 80 and $100{ }^{\circ} \mathrm{C}$ for $P$. pallida and $P$. juliflora, respectively.
\end{abstract}

KEYWORDS: Seed viability; germination; response to fire.

\section{INTRODUCCIÓN}

El género Prosopis está ampliamente distribuido en varios ecosistemas agroforestales, principalmente en climas semiáridos y áridos de América, Asia y África (Carevic, 2014) y en Oceanía como especies introducidas (Pasiecznik, Felker, Cruz, \& Cadoret, 2001). De las 44 especies de Prosopis, 30 crecen en Estados Unidos. Se supone que Argentina es el centro de origen del género Prosopis, y eso podría explicar el hecho de que este país tiene la mayor variabilidad de especies. Las especies de Prosopis son de gran valor ecológico y social, ya que proporcionan bienes como leña, alimentos, piensos para el ganado y medicinas, así como servicios que actúan como estabilizadores de cuencas hidrográficas y secuestradores de nitrógeno y carbono (Cangiano, Cendoya, Risio-Allione, \& Bogino, 2020). No todas las especies de este género son de importancia económica, siendo muchas de ellas arbustos de valor limitado (Pasiecznik et al., 2001).

Los árboles del género Prosopis son resistentes a la sequía (Pedersen, 1980), a temperaturas extremas (Ortiz \& Cardemil, 2001) y tolerantes a la salinidad (Pedersen, 1980; Pasiecznik et al., 2001; Felker, Clark, Laag, \& Pratt, 1981). P. juliflora y P. pallida son capaces de sobrevivir y crecer en sitios pobres que pocas otras especies tolerarán (Pasiecznik et al., 2001). Además, según Villagra et al. (como se citó en Sciammaro, Ribotta, \& Puppo, 2016), son fácilmente adaptable a una gran diversidad climática, principalmente a climas subhúmedos y secos. 
El oxígeno, la temperatura, el agua y la luz son condiciones necesarias para que ocurra la germinación (Baskin \& Baskin, 2014; Bewley et al., 2013). El agua es uno de los factores ambientales que más influye en el proceso germinativo de las semillas, pues la rehidratación de los tejidos acelera las actividades metabólicas que resultan en el aporte de energía y nutrientes necesarios para la reanudación del crecimiento del eje embrionario, además de estar involucrado en todas las demás fases posteriores del metabolismo de la planta (Rego et al., 2011). La humedad y la temperatura son los factores más determinantes en el proceso de germinación, y cuando la humedad no es limitante, la tasa y el porcentaje de germinación dependen de la temperatura (Hadas, 2004).

La germinación de semillas de muchas especies creciendo en ecosistemas dependientes del fuego, se ve favorecida por una breve exposición a altas temperaturas (Cóbar-Carranza, García, Pauchard \& Peña, 2015). Los productos del fuego como el calor, el humo, las cenizas y madera carbonizada tienen una influencia física y fisiológica en la dormancia de semillas (Herrero, Martin \& Bravo, 2007).

Además del calor, existen otros tratamientos pregerminativos. Rodríguez Araujo, Pérez y Bonvissuto (2017), obtuvieron que la escarificación con ácido mejora eficazmente la germinación de semillas. El tiempo de exposición de la escarificación química con ácido sulfúrico influyó en la calidad de la germinación, es decir, la radícula óptima frente a la emergencia de los cotiledones. Este patrón también dependió del origen fitogeográfico de la especie.

Las semillas de Prosopis sp. necesitan tratamiento pre-germinativo para aumentar el porcentaje de germinación, así, Guerrero y López (como se cita en Ministerio de Agricultura, Ganadería, Acuacultura y Pesca [MAGAP], 2014), mencionan que en condiciones de vivero la semilla tarda entre 18 a 23 días para germinar, mientras que tratándolas con ácido sulfúrico por un lapso de 20 minutos o al remojarlas por un lapso de 24 horas, tardan en germinar ocho días.

En correspondencia con lo anteriormente planteado y considerando que las especies de P. pallida y $P$. juliflora se encuentran en la zona sur de la provincia de Manabí, tanto en el bosque seco tropical como en tierras de vocación forestal en las cuales es frecuente el uso del fuego en las actividades agropecuarias, el objetivo de esta investigación fue evaluar los efectos de la temperatura en la germinación de dichas especies como un indicador de su respuesta al fuego.

\section{DESARROLLO}

Materiales y métodos

\section{Ubicación del experimento y procedencia de las semillas}

El experimento se realizó en el Laboratorio de Bromatología de la Universidad Estatal del Sur de Manabí. Se utilizaron semillas $P$. pallida y $P$. juliflora procedentes de Rocafuerte, provincia de Manabí, las cuales una vez recolectadas, se colocaron en fundas herméticas y se conservaron a temperatura ambiente durante dos semanas antes de la realización del experimento.

\section{Evaluación de la viabilidad}

La evaluación de la viabilidad y el vigor de las semillas de las especies objeto de estudio se realizó a través de una prueba de germinación. Se utilizaron 60 semillas de cada especie, las cuales fueron tomadas al azar de las que posteriormente se utilizarían en el experimento. Para disminuir el tiempo de germinación se utilizó el tratamiento pregerminativo de imbibición en agua durante 24 horas, según se indica en el Programa de Incentivos para la Reforestación con 
Ramos-Rodríguez, M. P., Ugalde-Párraga, J. L., Medranda-Mendieta, J. A., Estévez-Valdés, I., Manríquez-Toala, T.

Fines Comerciales (MAGAP, 2014). Posteriormente se colocaron 3 repeticiones de 20 semillas cada una para las dos especies sobre papel absorbente colocado en bandejas de aluminio, el cual fue humedecido con agua destilada todos los días. La emergencia de la radícula fue el criterio utilizado para determinar si las semillas habían germinado. El periodo de observación, con registro diario, terminó 10 días después de que se registró la última semilla germinada. Los resultados obtenidos se expresaron como porcentaje de semillas germinadas (porcentaje de viabilidad). Se utilizó la Ecuación 1 (Barone, Duarte, \& Luna, 2016).

$$
P G=\left(\frac{\text { semthas germtrudas }}{\text { semthles prefitas a germtmax }}\right) * 100
$$

\section{Ensayos de germinación}

El ensayo utilizado para determinar el efecto de la temperatura sobre la germinación de P. pallida y $P$. juliflora consistió en colocar las semillas distribuidas uniformemente en bandejas de aluminio y someterlas a cinco tratamientos de temperatura en estufa eléctrica a $60,80,100,150$ y $200{ }^{\circ} \mathrm{C}$ durante tiempos de 0,$5 ; 1,0 ; 5,0$ y 10,0 minutos en cada caso. Una vez que las semillas recuperaron la temperatura ambiente se colocaron 3 repeticiones de 15 semillas de cada tratamiento en bandejas de aluminio sobre papel absorbente el cual fue humedecido con agua destilada todos los días. Otras tres repeticiones de igual número de semillas no se sometieron a ningún tratamiento térmico y se consideraron como control (Figura 1).

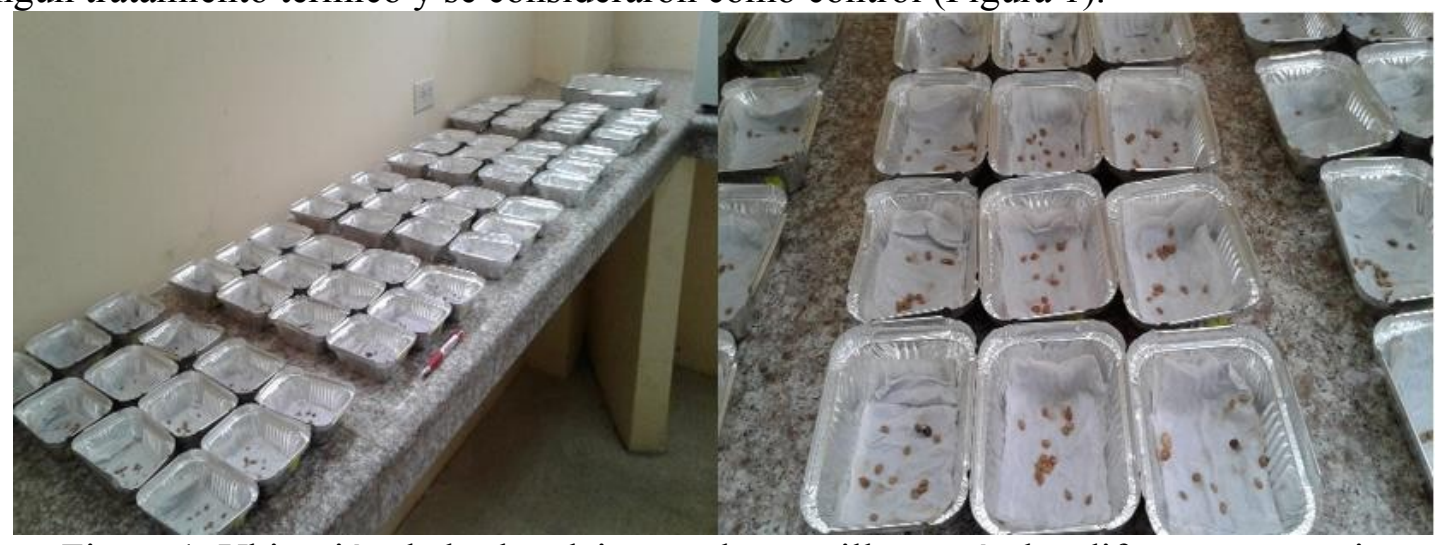

Figura 1. Ubicación de las bandejas con las semillas según los diferentes tratamientos

En el Laboratorio de Bromatología de la Universidad Estatal del Sur de Manabí las temperaturas promedio fueron de $25,82{ }^{\circ} \mathrm{C}$ durante el día y $18,52{ }^{\circ} \mathrm{C}$ por la noche. Durante el día la humedad relativa fue como promedio de $68,38 \%$ y durante la noche de $84,68 \%$.

La germinación se revisó diariamente, terminando este proceso 10 días después de que se registró la última semilla germinada. Se consideró la germinación fisiológica sensu stricto, es decir, extrusión de la radícula embrional a través de la cubierta seminal, con un largo mínimo de $2 \mathrm{~mm}$ (Madueño-Molina et al. 2006; Toniutti \& Fornasero, 2008). Con los datos obtenidos se determinó el porcentaje de germinación (PG) con la Ecuación 1 y el índice de velocidad de germinación (IVG) con la Ecuación 2 (Ranal \& García de Santana, 2006). Donde, $n i$ es el número de semillas germinadas en el intervalo de tiempo $t i$ y $\Sigma t i$ es el período en días desde la siembra hasta el día final de la experimentación.

24 UNESUM-Ciencias. Publicación cuatrimestral. Vol. 5, Año 2021, No. 6 (Especial Ingenierías) 
Publicación cuatrimestral. Vol. 5, No. 6 (Especial Ingenierías), Año 2021. Pág. 21-32 EFECTOS DE LA TEMPERATURA EN LA GERMINACIÓN DE SEMILLAS

$I V G=\Sigma_{\Sigma t i}^{m i}$

\section{Diseño Experimental y Procesamiento Estadístico}

Se trabajó con 40 tratamientos resultantes del arreglo trifactorial $2 \times 5 \times 4+1$, correspondiente a dos especies, cinco valores de temperaturas, cuatro tiempos de exposición y un testigo (Tabla 1), los cuales se asignaron a un diseño experimental completamente al azar con tres repeticiones, para un total de 120 unidades experimentales. En el caso de la prueba de viabilidad, se comprobó la normalidad de los datos con la prueba de Shapiro-Wilks obteniéndose en ambos caso valores de significación superiores a 0,05 . Posteriormente, se les aplicó una prueba paramétrica de $t$ de Student.

Tabla 1. Tratamientos utilizados en el experimento

\begin{tabular}{|c|c|c|c|}
\hline Tratamientos & Descripción & Tratamientos & Descripción \\
\hline $\mathrm{T} 1$ & P. pallida $+60{ }^{\circ} \mathrm{C}+30 \mathrm{~s}$ & $\mathrm{~T} 21$ & P. juliflora $+60{ }^{\circ} \mathrm{C}+30 \mathrm{~s}$ \\
\hline $\mathrm{T} 2$ & P. pallida $+60{ }^{\circ} \mathrm{C}+60 \mathrm{~s}$ & $\mathrm{~T} 22$ & P. juliflora $+60{ }^{\circ} \mathrm{C}+60 \mathrm{~s}$ \\
\hline $\mathrm{T} 3$ & P. pallida $+60{ }^{\circ} \mathrm{C}+300 \mathrm{~s}$ & $\mathrm{~T} 23$ & P. juliflora $+60^{\circ} \mathrm{C}+300 \mathrm{~s}$ \\
\hline $\mathrm{T} 4$ & P. pallida $+60{ }^{\circ} \mathrm{C}+600 \mathrm{~s}$ & $\mathrm{~T} 24$ & P. juliflora $+60{ }^{\circ} \mathrm{C}+600 \mathrm{~s}$ \\
\hline T5 & P. pallida $+80^{\circ} \mathrm{C}+30 \mathrm{~s}$ & $\mathrm{~T} 25$ & P. juliflora $+80^{\circ} \mathrm{C}+30 \mathrm{~s}$ \\
\hline T6 & P. pallida $+80{ }^{\circ} \mathrm{C}+60 \mathrm{~s}$ & $\mathrm{~T} 26$ & P. juliflora $+80{ }^{\circ} \mathrm{C}+60 \mathrm{~s}$ \\
\hline $\mathrm{T} 7$ & P. pallida $+80{ }^{\circ} \mathrm{C}+300 \mathrm{~s}$ & $\mathrm{~T} 27$ & P. juliflora $+80{ }^{\circ} \mathrm{C}+300 \mathrm{~s}$ \\
\hline $\mathrm{T} 8$ & P. pallida $+80{ }^{\circ} \mathrm{C}+600 \mathrm{~s}$ & $\mathrm{~T} 28$ & P. juliflora $+80{ }^{\circ} \mathrm{C}+600 \mathrm{~s}$ \\
\hline T9 & P. pallida $+100{ }^{\circ} \mathrm{C}+30 \mathrm{~s}$ & $\mathrm{~T} 29$ & P. juliflora $+100{ }^{\circ} \mathrm{C}+30 \mathrm{~s}$ \\
\hline $\mathrm{T} 10$ & P. pallida $+100{ }^{\circ} \mathrm{C}+60 \mathrm{~s}$ & $\mathrm{~T} 30$ & P. juliflora $+100^{\circ} \mathrm{C}+60 \mathrm{~s}$ \\
\hline $\mathrm{T} 11$ & P. pallida $+100{ }^{\circ} \mathrm{C}+300 \mathrm{~s}$ & $\mathrm{~T} 31$ & P. juliflora $+100{ }^{\circ} \mathrm{C}+300 \mathrm{~s}$ \\
\hline $\mathrm{T} 12$ & P. pallida $+100{ }^{\circ} \mathrm{C}+600 \mathrm{~s}$ & T32 & P. juliflora $+100^{\circ} \mathrm{C}+600 \mathrm{~s}$ \\
\hline $\mathrm{T} 13$ & P. pallida $+150{ }^{\circ} \mathrm{C}+30 \mathrm{~s}$ & $\mathrm{~T} 33$ & P. juliflora $+150{ }^{\circ} \mathrm{C}+30 \mathrm{~s}$ \\
\hline $\mathrm{T} 14$ & P. pallida $+150{ }^{\circ} \mathrm{C}+60 \mathrm{~s}$ & T34 & P. juliflora $+150{ }^{\circ} \mathrm{C}+60 \mathrm{~s}$ \\
\hline T15 & P. pallida $+150{ }^{\circ} \mathrm{C}+300 \mathrm{~s}$ & T35 & P. juliflora $+150{ }^{\circ} \mathrm{C}+300 \mathrm{~s}$ \\
\hline T16 & P. pallida $+150{ }^{\circ} \mathrm{C}+600 \mathrm{~s}$ & T36 & P. juliflora $+150{ }^{\circ} \mathrm{C}+600 \mathrm{~s}$ \\
\hline $\mathrm{T} 17$ & P. pallida $+200{ }^{\circ} \mathrm{C}+30 \mathrm{~s}$ & T37 & P. juliflora $+200{ }^{\circ} \mathrm{C}+30 \mathrm{~s}$ \\
\hline $\mathrm{T} 18$ & P. pallida $+200{ }^{\circ} \mathrm{C}+60 \mathrm{~s}$ & $\mathrm{~T} 38$ & P. juliflora $+200{ }^{\circ} \mathrm{C}+60 \mathrm{~s}$ \\
\hline T19 & P. pallida $+200{ }^{\circ} \mathrm{C}+300 \mathrm{~s}$ & T39 & P. juliflora $+200{ }^{\circ} \mathrm{C}+300 \mathrm{~s}$ \\
\hline $\mathrm{T} 20$ & P. pallida $+200{ }^{\circ} \mathrm{C}+600 \mathrm{~s}$ & $\mathrm{~T} 40$ & P. juliflora $+200{ }^{\circ} \mathrm{C}+600 \mathrm{~s}$ \\
\hline Control-1 & Condiciones ambientales & Control-2 & Condiciones ambientales \\
\hline
\end{tabular}

Los datos de porcentajes de germinación para la mayoría de los tratamiento no siguieron una distribución normal al aplicar la prueba de Shapiro-Wilks, por lo que los mismos fueron transformados sumándole a cada uno 10 unidades y posteriormente determinando el logaritmo en base 10. La posible existencia de diferencia entre las medias se determinó con un análisis de varianza trifactorial y la diferencia entre pares de medias con la prueba de Tukey. En todos los casos se utilizó un valor crítico de $\mathrm{p} \leq 0,05$. El procesamiento se realizó con el programa SPSS Statistics for Windows, Versión 22.0 (IBM Corp., 2013). 
Ramos-Rodríguez, M. P., Ugalde-Párraga, J. L., Medranda-Mendieta, J. A., Estévez-Valdés, I., Manríquez-Toala, T.

\section{Resultados}

\section{Viabilidad de las semillas de P. pallida y P. juliflora}

La prueba de germinación confirmó la viabilidad de las semillas. El porcentaje de germinación después de 22 días fue para el $P$. pallida y el $P$. juliflora de $81,67 \pm 7,63 \%$, y 80,00 $\pm 5,00 \%$; respectivamente. Según la prueba $t$ de Student $(-0,316)$ los resultados no mostraron diferencias significativas entre las especies respecto al porcentaje de germinación $(\mathrm{p}=0,768)$.

\section{Porcentaje de germinación}

A partir del tratamiento que incluía la temperatura de $100{ }^{\circ} \mathrm{C}$ y 30 segundos de exposición (T9 para $P$. pallida y T29 para $P$. juliflora) no hubo germinación en ninguna de las dos especies, por eso en los análisis no se consideran los tratamientos posteriores a estos. La Figura 2 muestra la distribución de los porcentajes de germinación de las dos especies según los tratamientos. En todos los casos los porcentajes son bajos. No obstante, para todos los tratamientos la especie $P$. pallida mostró mayores porcentajes de germinación que $P$. juliflora. Sin embargo, cuando las semillas fueron sometidas a temperatura de $80{ }^{\circ} \mathrm{C}$ tiempos de 300 y 600 segundos y de $100{ }^{\circ} \mathrm{C}$ por 60 segundos, solo existió germinación en el caso de la especie $P$. juliflora.

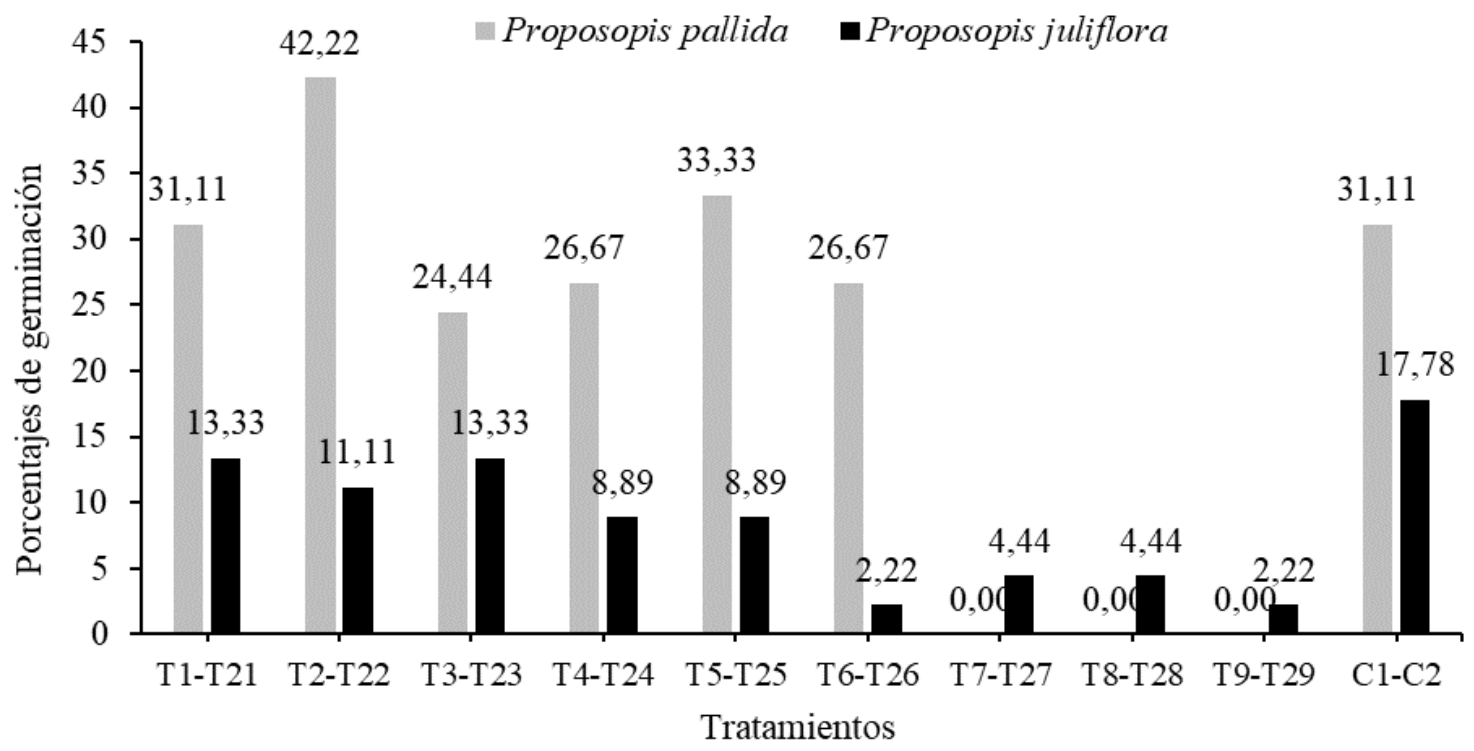

Figura 2. Distribución de los porcentajes de germinación de las dos especies según los tratamientos

El análisis de varianza trifactorial mostró que existieron diferencias estadísticas significativas entre especies, temperaturas, tiempos de exposición y tratamientos $(\mathrm{p} \leq 0,05)$; por su parte, las interacciones no mostraron diferencia significativa $(\mathrm{p} \leq 0,05)$ (Tabla 2$)$.

Tabla 2. Análisis de Varianza para el porcentaje de germinación de semillas de P. pallida y $P$. juliflora bajo diferentes tratamientos de temperaturas y tiempos de exposición a las mismas

\begin{tabular}{llll}
$\begin{array}{c}\text { Grados de } \\
\text { libertad }\end{array}$ & $\begin{array}{l}\text { Cuadrados } \\
\text { medios }\end{array}$ & F & P \\
\hline 19 & 0,167 & 7,771 & 0,00 \\
\hline
\end{tabular}

26 UNESUM-Ciencias. Publicación cuatrimestral. Vol. 5, Año 2021, No. 6 (Especial Ingenierías) 
Publicación cuatrimestral. Vol. 5, No. 6 (Especial Ingenierías), Año 2021. Pág. 21-32 EFECTOS DE LA TEMPERATURA EN LA GERMINACIÓN DE SEMILLAS

\begin{tabular}{lllll}
\hline Interceptación & 1 & 82,594 & 3847,269 & $\begin{array}{l}0 \\
0,00 \\
0\end{array}$ \\
Especies & 1 & 0,254 & 11,849 & 0,00 \\
Temperaturas & 2 & 0,661 & 30,786 & $\begin{array}{l}0,00 \\
0\end{array}$ \\
Tiempos & 3 & 0,138 & 6,440 & 0,00 \\
Especie*Temperatura & 2 & 0,109 & 5,078 & 1 \\
Especie*Tiempo & 3 & 0,127 & 5,896 & 1 \\
Temperatura*Tiempo & 3 & 0,069 & 3,211 & 0,00 \\
Interacciones & & & & 0,03 \\
Error & 3 & 0,048 & 2,244 & 3 \\
\hline
\end{tabular}

La mayor respuesta germinativa la mostraron las semillas de $P$. pallida sometidas a la temperatura de $60{ }^{\circ} \mathrm{C}$ un tiempo de 60 segundos. El P. juliflora presentó los mayores valores para la temperatura de $60{ }^{\circ} \mathrm{C}$ con tiempos de 30 y 300 segundos, aunque en ambos casos inferior al testigo. En sentido general, a medidas que aumentó tanto la temperatura como el tiempo de exposición, disminuyó el porcentaje de germinación (Tabla 3).

Tabla 3. Porcentaje de germinación promedio \pm desviación estándar (ds) y resultados de la prueba de comparación de medias de Tukey para las semillas de $P$. pallida y P. juliflora

\section{Tratamientos Germinación}

$\% \pm \mathbf{d s}$

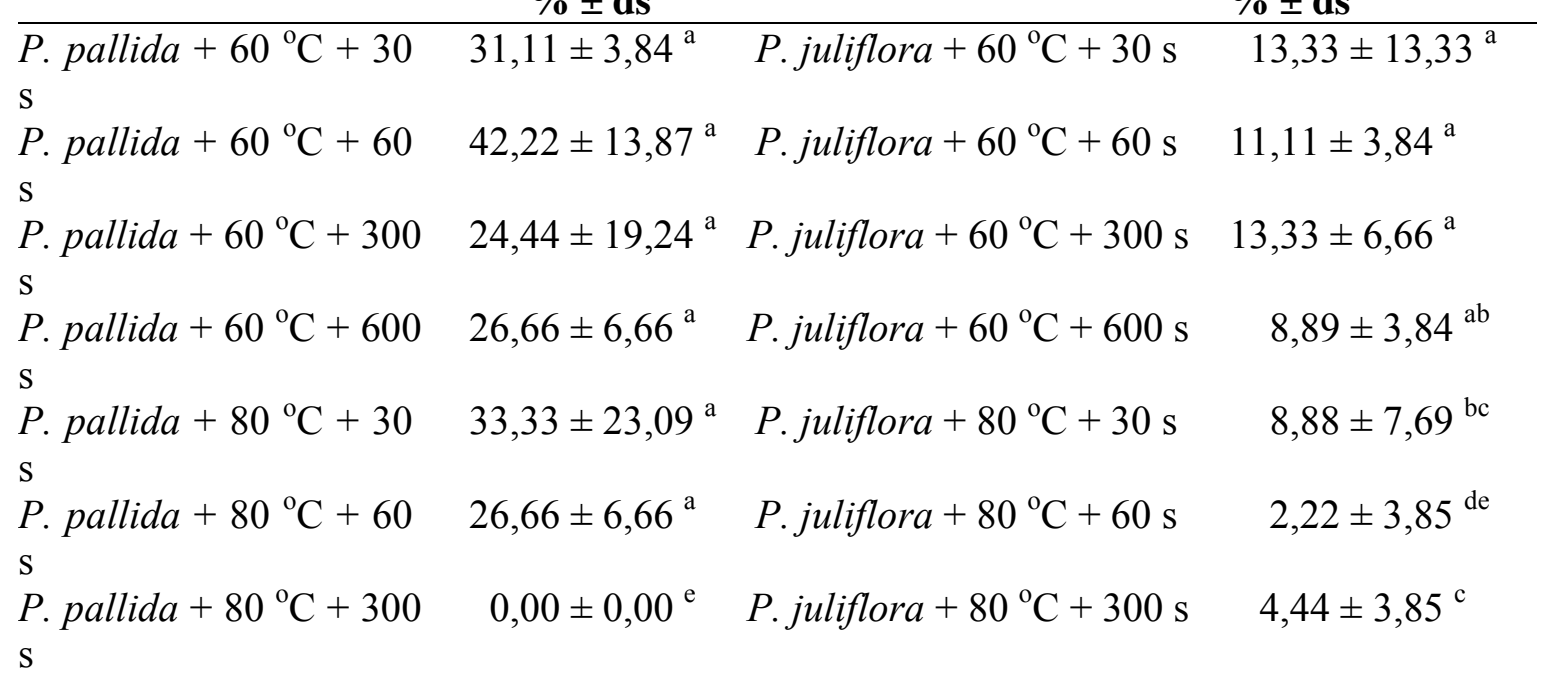


Ramos-Rodríguez, M. P., Ugalde-Párraga, J. L., Medranda-Mendieta, J. A., Estévez-Valdés, I., Manríquez-Toala, T.

$$
\begin{array}{lccc}
\text { P. pallida }+80{ }^{\circ} \mathrm{C}+600 & 0,00 \pm 0,00^{\mathrm{e}} & P . \text { juliflora }+80{ }^{\circ} \mathrm{C}+600 \mathrm{~s} & 4,44 \pm 7,69^{\mathrm{cd}} \\
\mathrm{S} & & & \\
\text { P. pallida }+100{ }^{\circ} \mathrm{C}+30 & 0,00 \pm 0,00^{\mathrm{e}} & P . \text { juliflora }+100{ }^{\circ} \mathrm{C}+30 \mathrm{~s} & 2,22 \pm 3,85^{\mathrm{d}}
\end{array}
$$

$\mathrm{S}$

Control-1 $\quad 31,11 \pm 7,69^{\text {a }} \quad$ Control-2 $\quad 17,77 \pm 13,87^{\text {a }}$

Nota: Letras iguales indican similitud entre los tratamientos respecto al \% de germinación.

\section{Índice de velocidad de germinación}

El promedio acumulado para la velocidad de germinación de las semillas de $P$. pallida se muestra en las Figuras 3A y 3B. Las semillas que fueron expuestas a la temperatura de $60{ }^{\circ} \mathrm{C}$ durante $30 \mathrm{y}$ 60 segundos comenzaron a germinar al noveno día y las que se expusieron a esa temperatura 300 y 600 segundos lo hicieron al décimo día. Por su parte las semillas expuestas por 30 y 60 segundos a $80{ }^{\circ} \mathrm{C}$ iniciaron la germinación al onceno día de iniciado el experimento. En todos los casos, a partir del día 25 de iniciado el experimento, no hubo más germinación.
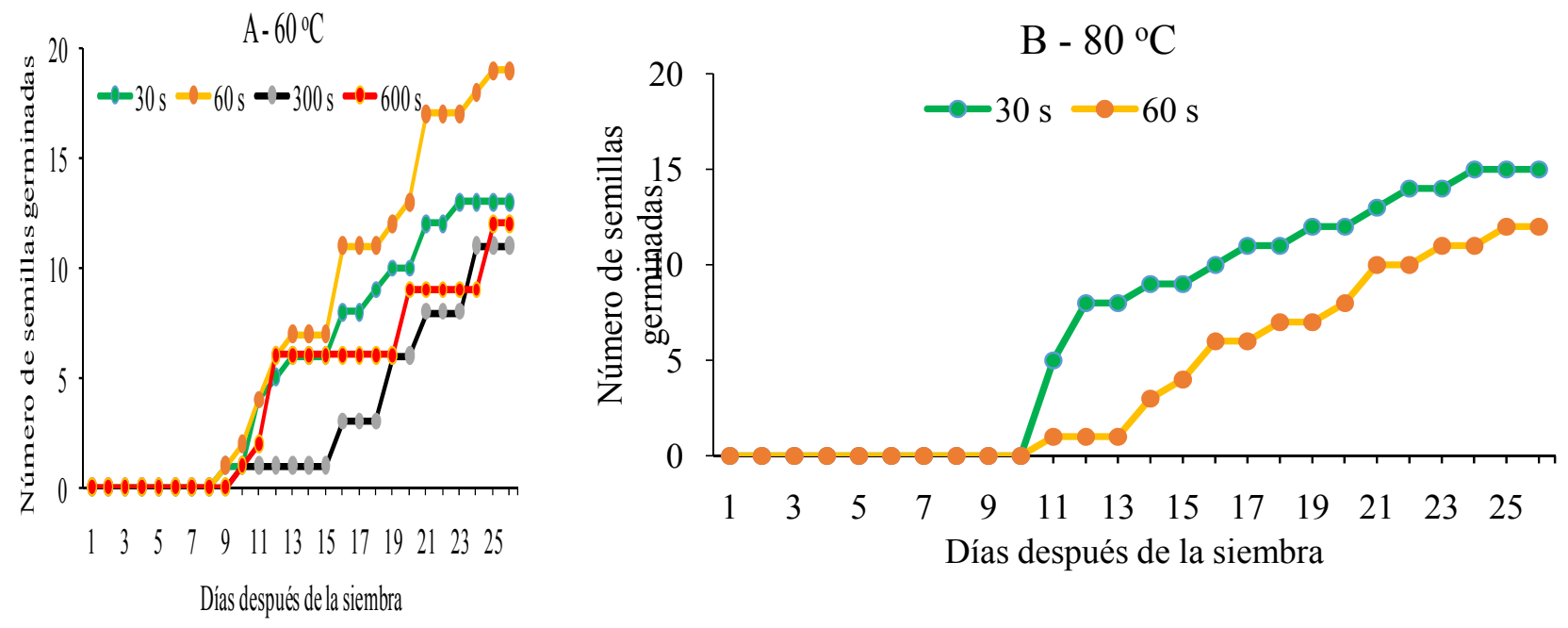

Figura 3. Promedio de la velocidad de germinación para las réplicas de 15 semillas de P. pallida. A: $60{ }^{\circ} \mathrm{C}$ para $30 \mathrm{~s}, 60 \mathrm{~s}, 300 \mathrm{~s}$ y $600 \mathrm{~s} . \mathrm{B}: 80^{\circ} \mathrm{C}$ para $30 \mathrm{~s}$ y $60 \mathrm{~s}$

El promedio acumulado para la velocidad de germinación de las semillas de $P$. juliflora se muestra en las Figuras 4A y 4B. Las semillas que fueron expuestas a la temperatura de $60{ }^{\circ} \mathrm{C}$ durante 30 y 60 segundos comenzaron a germinar al décimo día y las que se expusieron a esa temperatura 300 y 600 segundos lo hicieron a los 11 y 13 día. Por su parte las semillas expuestas por 30 y 60 segundos a $80{ }^{\circ} \mathrm{C}$ iniciaron la germinación a los 20 y 10 día de iniciado el experimento, respectivamente. Las expuestas a 300 y $600{ }^{\circ} \mathrm{C}$ iniciaron la germinación a los 20 y 11 días, respectivamente. 

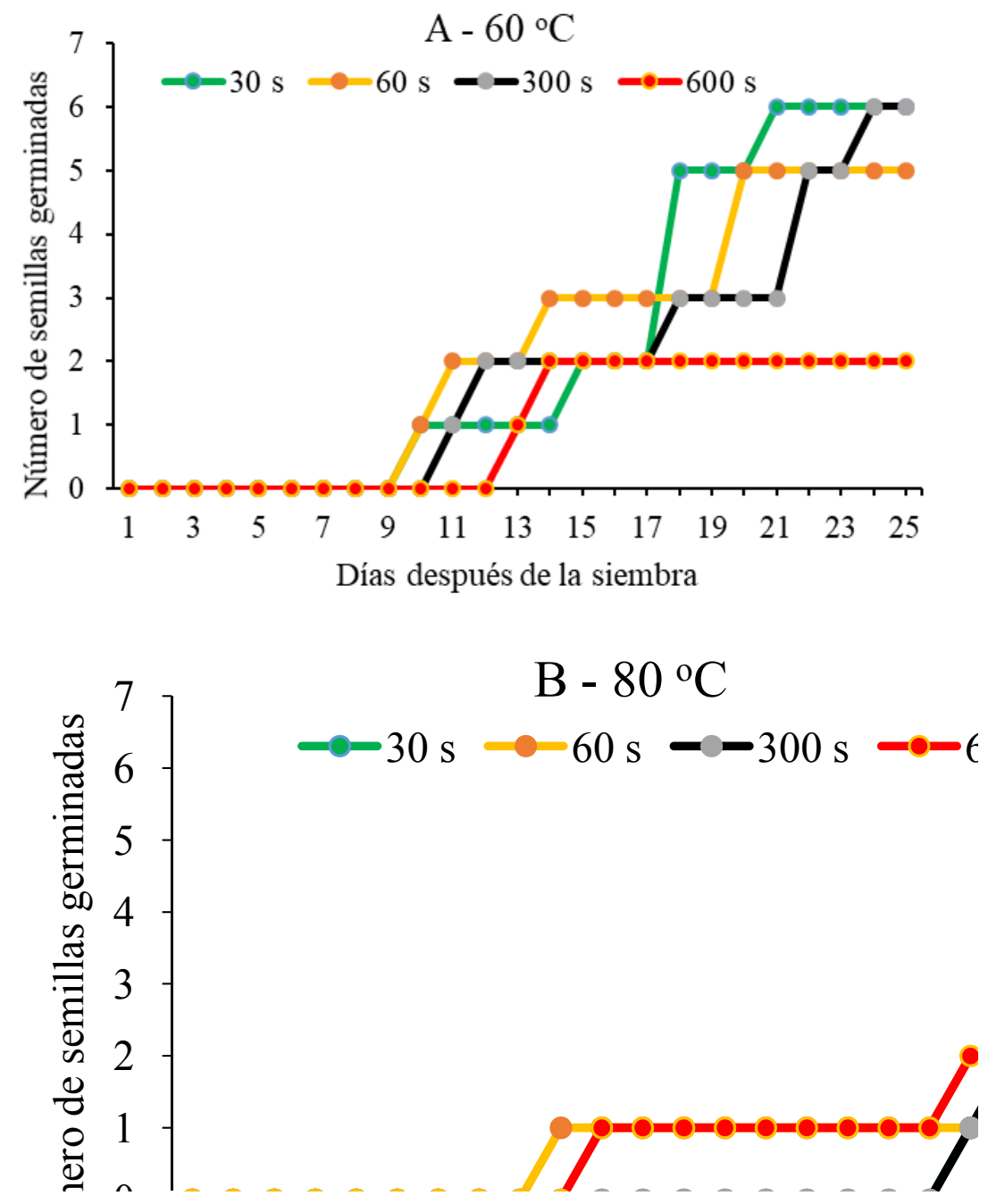

Figura 4. Promedio de la velocidad de germinación para las réplicas de 15 semillas de $P$. juliflora. A: $60{ }^{\circ} \mathrm{C}$ para $30 \mathrm{~s}, 60 \mathrm{~s}, 300 \mathrm{~s}$ y $600 \mathrm{~s}$. B: $80{ }^{\circ} \mathrm{C}$ para $30 \mathrm{~s}$ y $60 \mathrm{~s}$

\section{Discusión}

Valores similares a los utilizados en este experimento para las temperaturas y los tiempos de exposición han sido utilizados por investigadores tales como Herranz, Ferrandis, y MartínezSánchez (1998), con siete especies mediterráneas de leguminosas; Heras, Bonilla y Martínez (2006), en Pinus tropicalis Morelet y Pinus caribaea Morelet var. caribaea en Cuba; y CóbarCarranza, García, Pauchard y Peña (2015), con la especie invasora Pinus contorta y dos especies nativas del sur de Chile.

En esta investigación a partir del tratamiento que incluía la temperatura de $100{ }^{\circ} \mathrm{C}$ y 30 segundos de exposición (T9 para $P$. pallida y T29 para $P$. juliflora) no hubo germinación en ninguna de las dos especies, por eso en los análisis no se consideran los tratamientos posteriores a estos. La distribución de los porcentajes de germinación de las dos especies según los tratamientos en 
Ramos-Rodríguez, M. P., Ugalde-Párraga, J. L., Medranda-Mendieta, J. A., Estévez-Valdés, I., Manríquez-Toala, T.

todos los casos es baja. No obstante, para todos los tratamientos la especie P. pallida mostró mayores porcentajes de germinación que el $P$. juliflora. Sin embargo, cuando las semillas fueron sometidas a temperatura de $80{ }^{\circ} \mathrm{C}$ tiempos de 300 y 600 segundos y de $100{ }^{\circ} \mathrm{C}$ por 60 segundos, solo existió germinación en el caso de la especie $P$. juliflora.

Para el caso de Atriplex canescens inició la germinación alrededor del tercer día, concluyendo alrededor del día 15 , mostrando la mayor germinación $(17 \%)$ a $26^{\circ} \mathrm{C}$, con escarificación y sin fungicida. En los tratamientos $26^{\circ} \mathrm{C}, \mathrm{SE}, \mathrm{CF}$ y $30^{\circ} \mathrm{C}, \mathrm{SE}, \mathrm{CF}$ no hubo germinación y, en el resto de los tratamientos, la germinación fue inferior a 10\%. En general, la baja germinación de esta especie puede atribuirse a que las semillas del género Atriplex presentan un marcado estado de latencia, siendo esto una desventaja para su uso inmediato, ya que requieren de tratamientos como remojo en agua por 48 horas o escarificación (Contreras et al. 2012) para aumentar su germinación. Para Frankenia gypsophila, la germinación dio inicio en el primer día logrando más de un $80 \%$ de germinación para el día 5 , en siete de los ocho tratamientos. Lo anterior coincide con lo reportado por Flores et al. (2005) con Turbinicarpus swobodae y T. valdezianus que están adaptadas a germinar cuando ocurren eventos de lluvia, y aquí también se muestra esa adaptación en semillas frescas de F. gypsophila cuya respuesta de germinación, ante la presencia de humedad, fue muy rápida. La germinación de Machaeranthera pinnatifida, no fue más allá de un $8 \%$ con el tratamiento de $26^{\circ} \mathrm{C}$, porcentaje que alcanzó al día 9 y ya no aumentó en el resto del mes.

Las semillas que fueron expuestas a la temperatura de $60{ }^{\circ} \mathrm{C}$ durante 30 y 60 segundos comenzaron a germinar al noveno día y las que se expusieron a esa temperatura 300 y 600 segundos lo hicieron al décimo día. Por su parte las semillas expuestas por 30 y 60 segundos a 80 ${ }^{\circ} \mathrm{C}$ iniciaron la germinación al onceno día de iniciado el experimento. En todos los casos, a partir del día 25 de iniciado el experimento, no hubo más germinación.

De acuerdo a la hipótesis de Venable y Lawlor (1980), las semillas de especies anuales tienen germinabilidad más baja que las especies perennes. Los resultados también contrastan con lo expuesto por Jurado y Westoby (1992), quienes mencionan que una baja germinabilidad suele estar asociada con una rápida y media velocidad de germinación, mientras que una alta germinabilidad tiende a estar asociada con media y lenta velocidad de germinación. Para las especies analizadas en esta investigación encontramos que la alta germinabilidad estuvo asociada a rápida velocidad de germinación, mientras que la baja germinabilidad tuvo velocidades medias y lentas de germinación.

\section{AGRADECIMIENTOS}

A la Universidad Estatal del Sur de Manabí por financiar el proyecto de investigación intitulado "Incendios de vegetación en la provincia de Manabí, Ecuador (Primera Parte)" en el marco del cual se desarrolló la presente investigación.

\section{CONCLUSIONES}

Las semillas de $P$. pallida y $P$. juliflora utilizadas en la investigación eran viables, alcanzando ambas especies porcentajes de germinación aproximadamente del 80,00\% después de 22 días. El porcentaje de germinación de semillas de las especies $P$. pallida y $P$. juliflora es afectado tanto por el aumento de la temperatura como por el tiempo de exposición. No obstante, pudieran 
germinar después de la ocurrencia de fuegos de baja intensidad que generen temperaturas de hasta $80{ }^{\circ} \mathrm{C}$ para el caso de $P$. pallida y de $100{ }^{\circ} \mathrm{C}$ para el caso de $P$. juliflora.

El índice de velocidad de germinación de semillas de las especies $P$. pallida y $P$. juliflora en promedio acumulado reflejaron que a partir de los $60{ }^{\circ} \mathrm{C}$ y 30 segundos en ambas especies la germinación comenzó entre los días nueve y 20.

\section{REFERENCIAS BIBLIOGRÁFICAS}

Barone, J., Duarte, E., \& Luna, C. (2016). Determinación de la eficacia de métodos de evaluación de calidad de semillas de especies forestales nativas de la Selva Atlántica. Quebracho - Revista de Ciencias Forestales, 24(1,2), 70-80. Recuperado de https://fcf.unse.edu.ar/archivos/quebracho/v24n2a03-211.pdf

Cangiano, L. M., Cendoya, A. M., Risio-Allione, L. V., \& Bogino, S. M. (2020). Dendroecology of Prosopis Species in the World: Secular Traces of Natural and Anthropic Events and Their Effects on Prosopis Growth. In Latin American Dendroecology (pp. 121-140). https://doi.org/10.1007/978-3-030-36930-9 1

Carevic, F. S. (2014). The role of ecophysiological studies in the genus Prosopis : implications for the conservation of drought-prone species. IDESIA, 32(4), 77-82. Recuperado de https://scholar.google.es/scholar?hl=es\&as sdt=0\%2C $5 \& q=T h e+$ role + of + ecophysiological + studies + in + the $+g$ enus + Prosopis $\% 3 \mathrm{~A}+$ implications + for + the + conservation + of + drought-prone + species + Rol $\& b t n G=$

Felker, P., Clark, P. R., Laag, A. E., \& Pratt, P. F. (1981). Salinity tolerance of the tree legumes: Mesquite (Prosopis glandulosa var. torreyana, $\mathrm{P}$. velutina and P. articulata) Algarrobo (P. chilensis), Kiawe (P. pallida) and Tamarugo (P. tamarugo) grown in sand culture on nitrogen-free media. Plant and Soil, 61(3), 311-317. https://doi.org/10.1007/BF02182012

Hadas, A. 2004. Seedbed preparation: The soil physical environment of germinating seeds. p. 3-49. In R.L. BenechArnold and R.A. San- chez (eds.). Handbook of Seed Physiology: Applications to Agriculture. Food Product Press, New York, USA. Recuperado de https://books.google.es/books?hl=es\&lr=\&id=80dZDwAAQBAJ\&oi=fnd\&pg=PT23\&dq=Seedbed + preparati on:+The+soil+physical+environment + of + germinating+seeds.\&ots=M1MtKGy3je\&sig=KDvPMJrMq1HeFm bJ-

$\underline{1 \mathrm{cv}} 91 \mathrm{tT} 7 \mathrm{Ng} \# \mathrm{v}=$ onepage \&q $=$ Seedbed $\% 20$ preparation $\% 3 \mathrm{~A} \% 20 \mathrm{The} \% 20$ soil $\% 20$ physical $\% 20$ environment $\% 20$ of $\% 20$ germinating $\% 20$ seeds.\&f $=$ false

IBM Corp. (2013). IBM SPSS Statistics for Windows, Version 22.0. IBM Corp., Armonk, NY: IBM Corp.

Ortiz, C., \& Cardemil, L. (2001). Heat-shock responses in two leguminous plants : a comparative study. Journal of Experimental Botany, 52(361), $1711 \pm 1719 . \quad$ Recuperado de https://watermark.silverchair.com/521711.pdf?token=AQECAHi208BE49Ooan9kkhW_Ercy7Dm3ZL_9Cf3q fKAc485ysgAAAqYwggKiBgkqhkiG9w0BBwagggKTMIICjwIBADCCAogGCSqGSIb3DQEHATAeBglgh kgBZQMEAS4wEQQM8cprlaft61YYm5p_AgEQgIICWeg9fBAKZCmZqKy34bJYdV8LBP6gbaKfCmkziHJOPmRT2Ia

Pasiecznik, N. M., Felker, P., Cruz, G., \& Cadoret, K. (2001). The Prosopis juliflora-Prosopis pallida Complex : (p. 172). p. 172. HDRA Coventry UK.

Pedersen, B. O. (1980). A note on the genus. International Tree Crops Journal, 1(2-3), 113-123. https://doi.org/10.1080/01435698.1980.9752721

Ranal, M. A., \& García de Santana, D. (2006). How and why to measure the germination process? Revista Brasileira de Botanica, 29(1), 1-11. Recuperado de https://www.scielo.br/pdf/rbb/v29n1/a02v29n1.pdf

Rego, S. S., Ferreira, M. M., Nogueira, A. C., Grossi, F., Sousa, R. K. de, Brondani, G. E., ... Silva, A. L. L. da. (2011). Estresse hídrico e salino na germinação de sementes de Anadenanthera colubrina (Veloso) brenan. Journal of Biotechnology and Biodiversity, 2(4), 37-42. https://doi.org/10.20873/jbb.uft.cemaf.v2n4.rego 
Ramos-Rodríguez, M. P., Ugalde-Párraga, J. L., Medranda-Mendieta, J. A., Estévez-Valdés, I., Manríquez-Toala, T.

Rodríguez Araujo, M. E., Pérez, D. R., \& Bonvissuto, G. L. (2017). Seed germination of five Prosopis shrub species (Fabaceae-Mimosoideae) from the Monte and Patagonia phytogeographic provinces of Argentina. Journal of Arid Environments, 147, 159-162. https://doi.org/10.1016/j.jaridenv.2017.07.019

Sciammaro, L. P., Ribotta, D. P., \& Puppo, M. C. (2016). Traditional Foods. In Traditional Foods (pp. 209-216). https://doi.org/10.1007/978-1-4899-7648-2

32 UNESUM-Ciencias. Publicación cuatrimestral. Vol. 5, Año 2021, No. 6 (Especial Ingenierías) 